\title{
Prise en charge des body-packers
}

\section{Management of « Body-Packers »}

\author{
G. Schmit · F. Bou Abdallah - J. Vanhaebost $\cdot$ P. Hantson
}

Reçu le 18 janvier 2018; accepté le 10 avril 2018

(C) SRLF et Lavoisier SAS 2018

Résumé Aujourd'hui, le body-packing ou transport in corpore de substances illicites est le mode de transport de drogues le plus répandu en Europe. Il porte essentiellement sur la cocaïne et l'héroïne, bien que toute autre substance puisse être concernée dans une moindre mesure. Les passeurs («mules») sont identifiés par les services de police et de douane sur la base de plusieurs indices permettant de les suspecter, comme l'origine des vols long-courriers, l'abstinence alimentaire, etc. La sensibilité du dépistage urinaire et le diagnostic reposent essentiellement sur l'imagerie abdominale, de préférence l'examen tomodensitométrique lorsqu'il est disponible. Les passeurs sont ensuite placés sous surveillance médicale. Celle-ci n'est pas standardisée, mais devrait durer au moins six heures, avec une attention particulière aux paramètres hémodynamiques et neurologiques. Le risque de rupture des paquets est principalement lié à la qualité de l'emballage. Le traitement est essentiellement conservateur. Les complications restent rares, et le recours à la chirurgie s'avère nécessaire en cas de signes d'intoxication et/ou d'occlusion digestive.

Mots clés Body-packing · Diagnostic · Complications · Surveillance $\cdot$ Thérapeutique

G. Schmit · J. Vanhaebost

Service d'anatomie pathologique, centre de médecine forensique, cliniques universitaires Saint-Luc, B-1200 Bruxelles, Belgique

F. Bou Abdallah

Département de médecine forensique,

faculté des sciences médicales,

Université libanaise, Had, Liban

P. Hantson $(\bowtie)$

Département des soins intensifs,

Université catholique de Louvain,

cliniques universitaires Saint-Luc, Bruxelles,

B-1200 Bruxelles, Belgique

e-mail : philippe.hantson@uclouvain.be

Louvain Center for Toxicology and Applied Pharmacology,

Université catholique de Louvain,

cliniques universitaires Saint-Luc,

B-1200 Bruxelles, Belgique
Abstract "Body-packing" refers to the internal concealment of drugs within the gastrointestinal tract or other orifices. Currently, it represents the most frequent mode of drug smuggling in Europe. Among the substances involved, packing with cocaine or heroin is of most concern. Individuals who refrain from eating or drinking during long-distance flights may be suspected of concealing drugs. The sensitivity of urinary toxicological screening is weak, and the diagnosis is mainly based on abdomen X-ray, or preferentially computed tomography examination. "Body-stuffers" are then kept under medical supervision, for a period of at least 6 hours, with a special attention to hemodynamic and neurological changes. The risk of leakage or rupture of the packets seems directly related to the number and quality of the layers used for packing. Supportive therapy is usually sufficient. Complications are rare, but urgent surgery is required in case of signs of systemic toxicity and/or gastrointestinal obstruction.

Keywords Body-packing · Diagnosis · Complications · Monitoring · Treatment

\section{Introduction}

Initialement décrit en 1973, le body-packing ou transport de stupéfiants in corpore, désigne la dissimulation par ingestion ou insertion dans le tractus gastro-intestinal de substances illégales [1]. Depuis lors, cette pratique connaît un essor important. En Europe, elle constitue aujourd'hui le mode le plus répandu de trafic de la cocaïne, avec comme principaux continents pourvoyeurs l'Amérique du Sud et l'Afrique du Nord. Le terme de body-packer est à différencier du bodystuffer ; ce dernier désigne la personne qui ingère une substance dans un but de dissimulation aux autorités.

Les substances illégales principalement concernées par le body-packing sont le cannabis, puis l'héroïne. Cependant, on peut également retrouver d'autres substances telles que la méthamphétamine, les substances cannabinoïdes, l'ecstasy ou encore l'acide lysergique (LSD). 
Le recours au body-packing n'est pas limité aux hommes. Cette pratique touche aujourd'hui les différents groupes démographiques : les femmes, y compris les femmes enceintes, les enfants, même les animaux comme des chiens ou des chameaux [2,3]. Les substances sont ingérées ou encore insérées par voie rectale, vaginale, ou même dans le conduit auditif externe [4-7]. Les body-packers, ou « mules », transportent habituellement jusqu'à un kilogramme de substances illicites, réparties en paquets de cinq à dix grammes chacun et de 3 à $20 \mathrm{~mm}$ de diamètre. Le nombre de paquets transportés est habituellement de 50 à 100 , mais ce nombre peut atteindre 500 [8-10].

Les techniques d'emballage employées actuellement utilisent des procédés de plus en plus sophistiqués. Les substances transportées sont enveloppées dans des capsules fabriquées à partir de cellophane, de latex, de ballons, de préservatifs, de biberons, de cire ou de rubans autoadhésifs [11].

On définit quatre types de paquets. Le type 1 est constitué uniquement d'une enveloppe formée de deux à quatre couches de latex, ce qui prédispose à un risque élevé de rupture. Le type 2, de taille plus grande, est plus fermement enveloppé et noué, avec cinq à sept couches de latex. Il est plus robuste et plus résistant à la dégradation. Le perfectionnement de ces paquets se traduit dans le type 3 , qui possède une couche de papier d'aluminium en supplément, diminuant la densité radiographique du paquet et compliquant sa détection. De plus, les paquets de type 3 les plus récents ont des dimensions et des poids standardisés qui reflètent un procédé mécanique de fabrication. Ils contiennent de la poudre fortement condensée et des enveloppes additionnelles de cire. Le type 4 consiste en une nouvelle forme de cocaïne industrialisée, préparée à partir d'hydrochloride de cocaïne dissoute dans une solution à base d'alcool. Ce type de paquet contient également des papiers d'aluminium, du papier plastique, du papier carbone ou d'autres matériaux pour diminuer la densité radiographique [12].

\section{Diagnostic}

Les passeurs, désignés sous le nom usuel de « mules », sont habituellement identifiés par les fonctionnaires de police, des douanes ou par le personnel navigant en raison d'un comportement suspect. Les hôtesses de l'air et les stewards les décrivent comme des passagers qui typiquement sur un vol long-courrier ne boivent ni ne mangent pratiquement rien, s'abstiennent de fréquenter les toilettes, ont un comportement bizarre, ne bougent quasiment pas tout le long du trajet, ou encore ont une haleine cétosique en raison du jeûne [13]. En pratique, les passeurs ingèrent avant leur départ des agents anticholinergiques et des agents constipants tels que le diphénoxylate ou le lopésramide afin de ralentir leur transit intestinal et empêcher l'évacuation de la drogue. Une fois dans le pays de destination et après le passage à la douane, les passeurs ingèrent alors des laxatifs ou ont recours à des lavements afin de récupérer la drogue $[14,15]$.

Les body-packers peuvent aussi être identifiés suite à la survenue de complications. On distingue les complications mécaniques (obstruction intestinale) des complications toxiques suite au passage systémique de la drogue (épilepsie, arrêt cardiaque, détresse respiratoire, rhabdomyolyse, insuffisance rénale aiguë) provoqué par la fuite ou la rupture du paquet [16]. Une fois arrêtés par les forces de l'ordre, ils sont la plupart du temps amenés à la section médicale ou médicojudiciaire de l'aéroport.

Le dépistage toxicologique urinaire n'est pas fiable, surtout s'il n'y a pas eu rupture des paquets de drogue. En effet, il ne se baserait que sur une éventuelle contamination de la face externe des paquets lors de leur préparation. Ainsi, la sensibilité du dépistage urinaire est de $48 \%$ chez les personnes asymptomatiques [17]. Une technique d'imagerie est donc le plus souvent effectuée pour confirmer une suspicion de transport intracorporel de substance illicite. La radiographie abdominale simple, de par sa rapidité, simplicité, faible coût et dose d'irradiation, est l'imagerie de première ligne pour une confirmation diagnostique. Sa sensibilité varie de 40 à $94 \%$ [18,19]. Quatre signes typiques ont été décrits sur la radiographie simple : le tic-tac sign (93\%) signifie l'identification d'opacités denses, homogènes, oblongues, uniformes le long du tractus gastro-intestinal [20]. Le doublecondom sign désigne la découverte d'un croissant aérien entourant une densité bien définie, traduisant une fine couche d'air emprisonnée entre les couches de latex ou de cellophane du paquet [4]. Le parallelism sign (27\%) fait référence à la découverte de multiples opacités alignées de façon parallèle entre elles le long du tractus intestinal [21]. Enfin, le signe de la rosette se réfère à l'air emprisonné entre les nœuds de latex des paquets fabriqués manuellement [22].

Cependant, la radiographie simple présente un nombre de faux positifs en raison notamment de la constipation, de la présence de calculs rénaux ou de calcifications intraabdominales diverses $[23,24]$. Les cas de faux négatifs sont aussi nombreux d'une part à cause des progrès dans l'emballage (types 3 et 4) qui en incorporant des matériaux en aluminium et en plastique réduit la densité du matériel transporté [12], et d'autre part suite au recours au transport de cocaïne sous forme liquide dans des paquets à densité similaire aux selles [25].

Pour ces raisons, la tomodensitométrie abdominale reste la modalité de choix avec une spécificité élevée et une sensibilité approchant les $100 \%$. Elle permet de déterminer avec précision le nombre et la localisation des paquets, de rechercher des signes de rupture intracorporelle ou des complications éventuelles, notamment mécaniques [26,27]. Cependant, le problème de l'irradiation soulève une question éthique importante puisque l'examen est effectué non pas 
dans un but thérapeutique, mais plutôt avec une visée diagnostique qui peut avoir une implication médicolégale. La tomodensitométrie est donc réservée aux personnes pour lesquelles la radiographie simple n'avait pas apporté de réponse concluante [28].

Afin de répondre à cette problématique, une étude comparative a été menée dans une série de 40 body-packers. Chaque body-packer admis au service de radiologie a bénéficié d'une tomodensitométrie standard et d'une tomodensitométrie à faible dose. La tomodensitométrie à faible dose s'est révélée être une méthode appropriée de détection des body-packers lorsque les paquets mesurent plus d'un centimètre, avec une spécificité de $100 \%$ et une sensibilité de $86 \%$. En cas de signes cliniques évoquant une rupture de paquets, la tomodensitométrie standard reste toutefois l'examen diagnostique de choix [29].

\section{Surveillance et prise en charge}

\section{Surveillance}

Le diagnostic posé, le plus souvent par les techniques radiologiques, le patient est alors transféré en milieu spécialisé pour la surveillance de l'exonération des paquets et la prévention ou le traitement d'éventuelles complications [30]. Le milieu d'observation peut varier selon les pays, les législations et les circonstances. Le degré de surveillance médicale peut être variable selon que le lieu désigné serait une unité médicojudiciaire spécialisée, un service d'urgences hospitalières ou l'infirmerie d'un établissement pénitentiaire. Il paraît logique de pouvoir disposer d'un électrocardiogramme de référence à l'admission du patient pour pouvoir juger de l'évolution en cas de complications [31]. La durée de surveillance hospitalière d'un patient asymptomatique n'est pas établie. Certains auteurs parlent d'une durée minimale de six heures pour les body-stuffers, durée qui ne peut pas être extrapolée aux body-packers [32]. Dans une série récente de 132 patients (mais seulement 36 body-packers avérés), la durée moyenne de séjour dans le service des urgences était de 51,2 heures [33]. La durée de séjour n'était pas influencée par le nombre de paquets, leur localisation initiale ou des symptômes d'hypertension artérielle ou de tachycardie modérée à l'admission. Dans une série de 1250 body-packers, seuls $46(4,5 \%)$ avaient été surveillés en milieu hospitalier, les autres n'ayant présenté aucune complication [34]. Un certain nombre de signes ou symptômes peuvent être peu spécifiques (tachycardie et hypertension modérée, douleur abdominale) ou tout simplement liés au stress induit par la situation [33]. Tout patient présentant des signes d'occlusion ou de toxicité systémique doit être admis dans une unité de surveillance intensive. Le taux de complications est faible lorsque le patient est hospitalisé dans un milieu adéquat avec une surveillance rapprochée (8/581 patients admis dans une unité médicojudiciaire) [35]. L'admission dans un milieu de réanimation d'un patient asymptomatique ne se justifie pas sur la seule base d'un test urinaire positif pour la cocaïne à l'admission, car il s'agit le plus souvent d'un reflet d'une contamination de la surface externe des paquets lors de leur préparation [36]. Par contre, la persistance d'une positivité du test après quatre jours ou le virage d'un test initialement négatif sont suggestifs d'une fissuration des paquets [35].

Une évaluation médicale est évidemment indispensable avant la mise en route de tout traitement évacuateur. Il faut $\mathrm{y}$ adjoindre une surveillance continue avec prise de paramètres pendant la phase d'élimination. Les deux substances les plus souvent impliquées dans le trafic in corpore, la cocaïne devançant l'héroïne, exposent de fait à des complications majeures en cas de rupture ou de fissuration d'un ou de plusieurs paquets. La rupture d'un seul paquet de cocaïne peut s'avérer mortelle, sachant qu'un paquet contient habituellement dix grammes de cocaïne pure [37]. Pour l'héroïne, il existe un antidote efficace, la naloxone, ce qui peut permettre d'élargir les indications d'un traitement conservateur.

\section{Traitement conservateur}

Dans sa forme la plus simple, le traitement consiste en la mise à la diète du passeur jusqu'à évacuation spontanée de la totalité des paquets sous l'influence du péristaltisme intestinal [11,38-41]. Le patient peut être encouragé à se mobiliser pour ne pas majorer une certaine paresse intestinale [2]. La stimulation du péristaltisme intestinal par l'utilisation de laxatifs est discutée, mais beaucoup d'équipes utilisent l'huile de paraffine éventuellement combinée à un antispasmodique (trimébutine par exemple) qui stimule la motilité intestinale [36,42]. Un procinétique de la vidange gastrique comme l'érythromycine ou le métoclopramide a également été proposé [42]. Certaines observations suggèrent que l'huile de paraffine pourrait endommager le latex qui recouvre habituellement les paquets, ce qui n'est pas confirmé par l'expérience d'autres équipes [43-45]. D'autres équipes encore préconisent l'usage de polyéthylène glycol (PEG 4000) dans le contexte d'une irrigation intestinale totale par un agent osmotique [46]. Aucune de ces techniques n'a été validée par des études de bon niveau d'évidence.

L'utilisation de charbon de bois par voie orale $(1 \mathrm{~g} / \mathrm{kg}$ toutes les quatre heures) n'est pas validée [47,48]. Elle ne se justifie pas en présence de paquets intacts, et son rôle préventif en cas de rupture inopinée d'un paquet de cocaïne paraît bien illusoire. La durée totale de la période d'évacuation peut s'échelonner sur trois à six jours [11]. La plupart des paquets sont éliminés dans un délai de 30 heures, même si certains body-packers ont pu ingérer des agents 
anticholinergiques ou des opiacés dans le but de ralentir le transit intestinal [34]. Un contrôle radiologique simple ou par tomodensitométrie permet de s'assurer de l'exonération complète.

Dans un article rapportant l'expérience de Hillingdon au Royaume-Uni, 56/65 (91,8 \%) des body-packers furent traités de façon conservative, et un seul patient a été opéré en raison d'une obstruction du grêle due à une absence de progression des paquets après cinq jours [38].

\section{Traitement chirurgical}

Le taux de recours à la laparotomie est très variable dans la littérature en raison de grandes variations dans les indications du traitement chirurgical. C'est ainsi que dans une série iranienne de 175 patients, $27 \%$ ont été traités chirurgicalement (dans $79 \%$ des cas pour des manifestations toxiques et dans $21 \%$ pour cause obstructive) [49]. Ce taux relativement élevé d'interventions chirurgicales en comparaison avec les données de la littérature peut s'expliquer d'une part par une proportion importante (un tiers) de patients se présentant volontairement à l'hôpital pour des manifestations toxiques, d'autre part par une moindre fiabilité des emballages [50].

Dans une importante série rétrospective portant sur 581 body-packers, 573 ont pu quitter l'unité médicojudiciaire après cinq jours suite au traitement conservateur, et seuls deux patients ont été opérés pour une obstruction [35]. Le retard de l'évacuation de paquets intragastriques ne constitue pas en soi une indication chirurgicale, et le traitement conservateur doit être privilégié. On pourrait émettre l'hypothèse que le risque de rupture des paquets augmente avec le temps, mais le traitement symptomatique a pu être poursuivi chez certains patients jusqu'à 17 jours, sans complications notables, même s'il a été suggéré qu'un délai de plus de cinq jours sans progression des paquets pourrait être une indication chirurgicale [34,41,45,51].

Les deux motifs principaux de recours à une technique chirurgicale sont l'existence d'une obstruction mécanique digestive (possible depuis l'œsophage jusqu'au rectum) ou la survenue de complications systémiques consécutives à la rupture ou à la fissuration de paquets $[34,38,48,51-56]$.

L'obstruction mécanique se produit habituellement aux points de rétrécissement anatomique (cardia, pylore, valvule iléocæcale) ou en raison d'une torsion intestinale entraînée par la masse des paquets ingérés [57] (Fig. 1). Dans une courte série autopsique, la plupart des paquets avaient été retrouvés dans l'estomac, le pylore constituant un obstacle difficilement franchissable pour des corps étrangers volumineux [58]. Parfois, l'occlusion basse est liée à la taille des paquets qui ont été introduits par voie intrarectale.

Différentes techniques chirurgicales ont été proposées $[51,59]$. Les paquets sont le plus souvent éliminés par une ou plusieurs entérotomies en fonction de leur nombre, ce qui

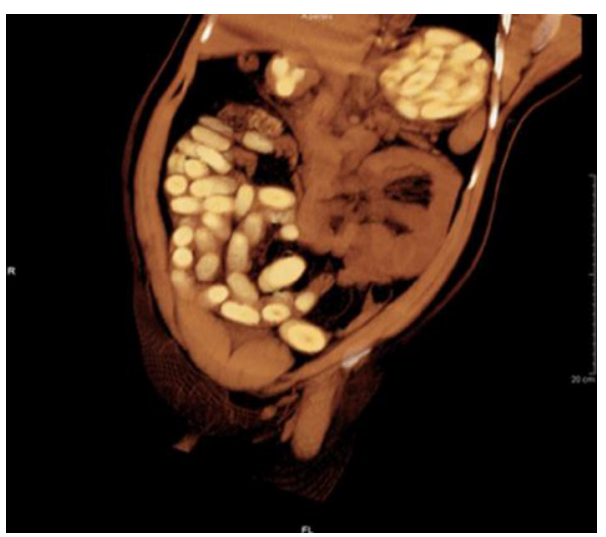

Fig. 1 Vue tomodensitométrique sagittale du thorax et de l'abdomen montrant la rétention d'une quantité importante de paquets intragastriques

peut accroître le risque postopératoire de fistule digestive $[52,60]$ (Fig. 2). Une autre technique consiste en la manipulation des paquets pour permettre leur migration et leur expulsion par l'anus [11,61]. Cette technique expose cependant au risque de rupture en cas d'empaquetage de mauvaise qualité. Le recours à l'endoscopie n'est pas recommandé en raison du risque de rupture [62,63]. L'endoscopie doit être considérée comme une technique de recours en cas de difficulté chirurgicale peropératoire ou pour vérifier pendant l'intervention l'élimination complète des paquets.

La chirurgie expose à des complications [55]. Dans une importante série monocentrique rapportant 70 patients opérés dans un contexte d'obstruction gastro-intestinale haute, la mortalité péri- et postopératoire était faible, de même que l'incidence de stomies. Cependant, l'incidence d'infections de plaies était importante $(32,9 \%)$ et semblait en relation avec le nombre d'entérotomies distales [64].
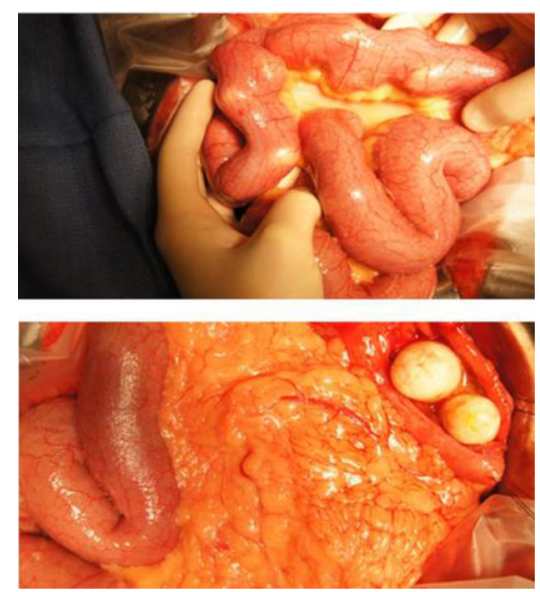

Fig. 2 Technique d'évacuation chirurgicale par palpation et migration des paquets au travers d'un ou de plusieurs orifices d'entérotomie 
L'incidence de rupture de paquets demeure très faible comme en témoigne une grande série collectée conjointement par les équipes françaises et allemandes à partir des passeurs de cocaïne identifiés dans les deux aéroports de Paris-Charles-de-Gaulle et de Francfort [65]. Entre 1985 et 2002, 4660 body-packers ont été identifiés. Cette série a montré que le recours à la chirurgie restait exceptionnel puisqu'il ne concernait que 20 patients $(0,4 \%)$ qui présentaient des symptômes compatibles avec une rupture des paquets et qui ont survécu. Cette intervention est la seule à pouvoir assurer la survie, puisque les 44 autres patients qui présentaient une complication comparable, mais qui n'ont pu être pris en charge à temps, sont décédés en l'absence d'intervention chirurgicale. Les causes de décès étaient soit neurologiques, soit cardiovasculaires.

La prise en charge des body-stuffers est comparable à celle des body-packers. La solidité des emballages est habituellement moins fiable dans ce cas de figure, mais la quantité totale dissimulée est plus faible et l'évacuation de plus courte durée.

\section{Traitement des manifestations toxiques}

La crainte principale est de voir apparaitre des manifestations toxiques de la cocaïne dans les sphères neurologique et cardiovasculaire. La principale complication neurologique est la survenue de crises d'épilepsie tonicocloniques généralisées, pouvant évoluer vers un état de mal épileptique. Ces crises justifient l'administration précoce d'une benzodiazépine qui réussit le plus souvent à contrôler la situation. Les effets cardiovasculaires sont secondaires à la stimulation adrénergique excessive (tachycardie, arythmies avec trouble de conduction intraventriculaire, hypertension, ischémie myocardique aiguë). Ces complications adrénergiques sont justifiables d'un traitement par un agent $\alpha$ - et $\beta$-bloquant, comme le labétolol, car le recours à un agent uniquement $\beta$-bloquant expose à un risque de vasoconstriction coronaire [2]. Des travaux font également mention de l'utilisation de dérivés nitrés ou d'inhibiteurs calciques, tant dans des modèles animaux qu'en clinique humaine. En cas d'élargissement du complexe QRS à l'électrocardiogramme par effet stabilisant de membrane, l'administration de bicarbonate de sodium molaire est justifiée, par palier de $100 \mathrm{mmol}$ jusqu'à normalisation du complexe QRS et sous surveillance de la kaliémie. L'utilisation d'émulsion lipidique (lipid rescue therapy) a été tentée dans quelques observations mais n'est pas soutenue par des études validées [66].

La symptomatologie principale de l'intoxication par opiacés comporte la triade principale : altération de la conscience, dépression respiratoire et myosis serré. Chez le patient qui ne requiert pas d'intubation orotrachéale, l'injection intraveineuse de l'antagoniste des opioïdes, la naloxone, se justifie en respectant des paliers qui permettent l'obten- tion d'une fréquence respiratoire sans révéler une agitation trop importante.

L'exposition à des dérivés amphétaminiques est nettement moins fréquente et moins spectaculaire que celle à la cocaïne. Si des symptômes adrénergiques majeurs se développent, le traitement se rapproche de celui de l'intoxication à la cocaïne.

Quant à l'exposition au cannabis, seul un ralentissement mental non critique pourrait être observé, et un simple traitement symptomatique est indiqué.

\section{Devenir des paquets}

Le devenir des paquets de drogue évacués naturellement ou extraits par chirurgie va dépendre du milieu dans lequel se trouve le body-packer.

Si l'évacuation des paquets se fait en milieu carcéral, le magistrat en charge du dossier ordonne généralement la destruction des paquets après que certains d'entre eux aient été analysés afin d'identifier leur contenu. L'identification du contenu est primordiale sur le plan judiciaire en tant qu'élément de preuve de l'infraction pénale.

En milieu médical, en dehors de toute procédure judiciaire, le médecin n'est pas tenu de dénoncer les faits aux autorités judiciaires. Les paquets sont stockés de manière anonyme dans les services hospitaliers de pharmacie et régulièrement détruits après accord d'un magistrat.

\section{Conclusion}

Le taux de complications des patients admis à la suite d'une dissimulation in corpore de substances illicites reste très faible. Ces patients requièrent néanmoins une surveillance continue jusqu'à une évacuation complète des paquets qui peut durer plusieurs jours. Le traitement conservateur à base de laxatifs est habituellement suffisant. Cependant, en cas de rupture notamment de paquets de cocaïne, la symptomatologie neurologique et cardiovasculaire peut devenir explosive, et seul le traitement chirurgical permet de sauver le patient. Il faut rester attentif à la possibilité d'obstruction intestinale, surtout en cas de rétention intragastrique de plusieurs paquets.

Liens d'intérêts : Les auteurs déclarent ne pas avoir de lien d'intérêt

\section{Références}

1. Deitel M, Syed AK, (1973) Intestinal obstruction by an unusual foreign body. Can Mad Assoc J 109: 211-212 
2. Traub SJ, Hoffman RS, Nelson LS, (2003) Body packing-the internal concealment of illicit drugs. N Engl J Med 349: 2519-2526

3. Annotations, (1940) A new way of smuggling narcotics. Lancet 235: 464

4. Berger FH, Nieboer KH, Goh GS, Pinto A, Scaglione M, (2015) Body packing: a review of general background, clinical and imaging aspects. Radiol Med 120: 118-132

5. Cappelletti S, Piacentino D, Sani G, Bottoni E, Fiore PA, Aromatario M, Cialella C, (2016) Systematic review of the toxicological and radiological features of body packing. Int J Legal Med 130: 693-709

6. Chakrabarty A, Hydros S, Puliyel JM, (2006) Smuggling contraband drugs using paediatric "body packers". Arch Dis Child 91: 51

7. Cordero DR, Medina C, Helfgott A, (2006) Cocaine body packing in pregnancy. Ann Emerg Med 48: 323-325

8. Gherardi RK, Baud FJ, Leporc P, Marc B, Dupeyron JP, Diamant-Berger O, (1988) Detection of drugs in the urine of body-packers. Lancet 1: 1076-1078

9. Bulstrode N, Banks F, Shrotria S, (2002) The outcome of drug smuggling by 'body-packers' - the British experience. Ann R Coll Surg Engl 84: 35-38

10. van Geloven AA, van Lienden KP, Gouma DJ, (2002) Bodypacking - an increasing problem in The Netherlands: conservative or surgical treatment? Eur J Surg 168: 404-409

11. Pidoto RR, Agliata AM, Bertolini R, Mainini A, Rossi G, Giani G, (2002) A new method of packaging cocaine for international traffic and implications for the management of cocaine body packers. J Emerg Med 23: 149-153

12. Shahnazi M, Hassanian-Moghaddam H, Gachkar L, Ahmadi N, Zamani N, Bahrami-Motlagh H, Faghihi Langroudi T, Arjmand Shabestari A, Mohammad Alizadeh A, (2015) Comparison of abdominal computed tomography with and without oral contrast in diagnosis of body packers and body stuffers. Clin. Toxicol (Phila) 53: 596-603

13. Kucukmetin NT, Gucyetmez B, Poyraz T, Yildirim S, Boztas G, Tozun N, (2014) Foreign material in the gastrointestinal tract: cocaine packets. Case Rep Gastroenterol 8: 56-60

14. UNODC, (2013) World Drug Report 2013 (United Nations publication, Sales $\mathrm{n}^{\mathrm{o}}$ E.13.XI.6)

15. Khan FY, (2005) The cocaine 'body-packer' syndrome: diagnosis and treatment. Indian J Med Sci 59: 457-458

16. Cawich SO, Hassranah D, Pooran S, Dan D, Narayansingh V, (2015) Complicated acute appendicitis? An unusual differential. Trop Doct 45: 49-51

17. Bogusz MJ, Althoff H, Erkens M, Maier RD, Hofmann R, (1995) Internally concealed cocaine: analytical and diagnostic aspects. J Forensic Sci 40: 811-815

18. Yegane RA, Bashashati M, Hajinasrollah E, Heidari K, Salehi NA, Ahmadi M, (2009) Surgical approach to body packing. Dis Colon Rectum 52: 97-103

19. Hergan K, Kofler K, Oser W, (2004) Drug smuggling by body packing: what radiologists should know about it. Eur Radiol 14: 736-742

20. Pinto A, Reginelli A, Pinto F, Sica G, Scaglione M, Berger FH, Romano L, Brunese L, (2014) Radiological and practical aspects of body packing. Br J Radiol 87: 20130500

21. Niewiarowski S, Gogbashian A, Afaq A, Kantor R, Win Z, (2010) Abdominal X-ray signs of intra-intestinal drug smuggling. J Forensic Leg Med 17: 198-202

22. Walter BM, Martignoni M, Säckl J, Felgenhauer N, Eyer F, Tratzl V, Schmid RM, von Delius S, (2012) A curious case of body packing: impaction of cocaine capsules in a colostomy exit. Forensic Toxicol 30:199-201
23. Karhunen PJ, Suoranta H, Penttila A, Pitkaranta P, (1991) Pitfalls in the diagnosis of drug smuggler's abdomen. J Forensic Sci 36: 397-402

24. Wilgoren J, (1998) Misdiagnosis led to man's handcuffing, suit claims. New York Times, http://www.nytimes.com/1998/12/06/ nyregion/misdiagnosis-led-to-man-s-handcuffing-suit-claims. html. Accessed 17 April 2015

25. Burillo-Putze G, Becker LT, Rodríguez MG, Torres JS, Nogué S, (2012) Liquid cocaine body packers. Clin Toxicol (Phila) 50: 522-524

26. Poletti PA, Canel L, Becker CD, Wolff H, Elger B, Lock E, Sara$\sin$ F, Bonfanti MS, Dupuis-Lozeron E, Perneger T, Platon A, (2012) Screening of illegal intracorporeal containers ("body packing"): is abdominal radiography sufficiently accurate? A comparative study with low-dose CT. Radiology 265: 772-779

27. Sica G, Guida F, Bocchini G, Iaselli F, Iadevito I, Scaglione M, (2015) Imaging of drug smuggling by body packing. Semin Ultrasound CT MR 36: 39-47

28. Union E, (1996) Council Directive 96/29/Euratom of 13 May 1996 laying down basic safety standards for the protection of the health of workers and the general public against the dangers of ionizing radiation., Access via, http://europa.eu/legislation summaries/employment_and_social_policy/health_hygiene_ safety_at_work/c11142_en.htm

29. Bahrami-Motlagh H, Mahboubi-Fooladi Z, Salevatipour B, Hassanian-Moghaddam H, Mirhashemi SH, (2018) Comparision of low dose and standard dose abdominal CT scan in body stuffers. Clin Toxicol (Phila) 56: 348-354

30. Guinier D, Hubert N, Chopard JL, (2007) Transport de stupéfiants in corpore : problèmes médicochirurgicaux et médicolégaux. J Chir 144: 481-485

31. Ortega-Carnicer J, Gomez-Grande L, Ambros A, (2005) Electrocardiographic changes indicating surgery on cocaine bodypackers. Resuscitation 65: 373-374

32. Sporer KA, Firestone J, (1997) Clinical course of crack cocaine body stuffers. Ann Emerg Med 29: 596-601

33. Heymann-Maier L, Trueb L, Schmidt S, Carron PN, Hugli O, Heymann E, Yersin B, (2017) Emergency department management of body packers and body stuffers. Swiss Med Wkly 147: w14499

34. Mandava N, Chang RS, Wang JH, Bertocchi M, Yrad J, Allamaneni S, Aboian E, Lall MH, Mariano R, Richards N, (2011) Establishment of a definitive protocol for the diagnosis and management of body packers (drug mules). Emerg Med J 28: 98-101

35. de Prost N, Lefebvre A, Questel F, Roche N, Pourriat JL, Huchon G, Rabbat A, (2005) Prognosis of cocaine body-packers. Intensive Care Med 31: 955-958

36. Megarbane B, Ekherian JM, Couchard AC, Goldgran-Toledano D, Baud F, (2004) La chirurgie au secours des body-packers. Ann Fr Anesth Reanim 23: 495-498

37. Koehler SA, Ladham S, Rozin L, Shakir A, Omalu B, Dominick J, Wecht C, (2005) The risk of body-packing: a case of fatal cocaine overdose. Forensic Sci Int 151: 81-84

38. Beckley I, Ansari NAA, Khwaja HA, Mohsen Y, (2009) Clinical management of cocaine body packers: the Hillingdon experience. Can J Surg 52: 417-421

39. Das D, Ali B, (1996) Towards evidence based emergency medicine: BETs from the Manchester Royal Infirmary. Conservative management of asymptomatic cocaine body packers. Emerg Med J 38: 111-116

40. Aldrighetti L, Paganelli M, Giacomelli M, Villa G, Ferla G, (1996) Conservative management of cocaine-packet ingestion: experience in Milan, the main Italian smuggling center of South America cocaine. Panminerva Med 38; 11-16 
41. Glovinski PV, Lauritsen ML, Bay-Nielsen M, Branstrup B, Bisgaard T, (2013) Asymptomatic body packers should be treated conservatively. Dan Med J 60: A4723

42. Traub SJ, Su M, Hoffman RS, Nelson LS, (2003) Use of pharmaceutical promotility agents in the treatment of body packers. Am J Emerg Med 21: 511-512

43. Visser L, Stricker B, Hoogendoorn M, Vinks A, (1998) Do not give paraffin to packers. Lancet 352: 1352

44. Marc B, Baud F, (1999) Paraffin and body-packers. Lancet 353: 238-239

45. Veyrie N, Servajean S, Aissat T, Corigliano N, Angelakov C, Bouillot JL, (2008) Value of a systematic operative protocol for cocaine body packers. World J Surg 32: 1432-1437

46. Hoffman RS, Smilkstein MJ, Goldfrank LR, (1990) Whole bowel irrigation and the cocaine-body packer: a new approach to a common problem. Am J Emerg Med 8: 523-527

47. Tomaszewski C, McKinney $\mathrm{P}$, Phillips S, Brent J, Kulig K, (1993) Prevention of toxicity from oral cocaine by activated charcoal in mice. Ann Emerg Med 22: 1804-1806

48. Olmedo R, Nelson L, Chu J, Hoffman R, (2001) Is surgical decontamination definitive treatment of "body-packer"? Am J Emerg Med 19: 593-596

49. Alipour-Faz A, Shadnia S, Mirhashemi SH, Peyvandi M, Oroei M, Shafagh O, Peyvandi H, Peyvandi AA, (2016) Assessing the Epidemiological Data and Management Methods of Body Packers Admitted to a Referral Center in Iran. Medicine (Baltimore) 95: e3656

50. McCarron MM, Wood JD, (1983) The cocaine "body packer" syndrome. Diagnosis and treatment. JAMA 250: 1417-1420

51. Silverberg D, Menes T, Kim U, (2006) Surgery for "body packers" - a 15-year experience. World J Surg 30: 541-546

52. Beck NE, Hale E, (1993) Cocaine "body packers" Br J Surg 80: 1513-1516

53. Gherardi R, Marc B, Alberti X, Baud F, Diamant-Berger O, (1990) A cocaine body-packer with normal abdominal plain radiograms. Value of drug detection in urine and contrast study of the bowel. Am J Forensic Med Pathol 11: 154-157
54. Álvarez Llano L, Rey Valcalcel C, Al-Lal YM, Pérez Díaz MD, Stafford A, Turégano Fuentes F, (2014) The role of surgery in the management of "body packers". Eur J Trauma Emerg Surg 40: 351-355

55. de Bakker JK, Nanayakkara PW, Geeraedts LM, de Lange ES, Mackintosh MO, Bonjer HJ, (2012) Body packers: a plea for conservative treatment. Langenbecks Arch Surg 397: 125-130

56. Hantson P, Capron A, Wallemacq P, (2011) Toxicokinetics of cocaine and metabolites in a body-packer becoming symptomatic. J Forensic Leg Med 18: 385-387

57. Hantson P, Capron A, Maillart JF, (2014) Oesophageal and gastric obstruction in a cocaine body packer. J Forensic Leg Med 27: $62-64$

58. Heinemann A, Miyaishi S, Iwersen S, Shmoldt A, Püschel K, (1998) Body-packing as cause of unexpected sudden death. Forensic Sci Int 92: 1-10

59. Suarez CA, Arango A, Lester JL, (1997) Cocaine-condom ingestion. JAMA 238: 1391-1392

60. Lancashire MJ, Legg PK, Lowe M, Davidson SM, Ellis BW, (1988) Surgical aspects of international drug smuggling. $\mathrm{Br}$ Med J (Clin Res Ed) 296: 1035-1037

61. Utecht MJ, Stone AF, McCarron MM, (1993) Heroin body packers. J Emerg Med 11: 33-40

62. Jones OM, Shorey BA, (2002) Body packers: grading of risk as a guide to management and intervention. Ann R Coll Surg Engl 84: $131-132$

63. Wong GCK, Lai KK, Chung CH, (2005) Management of body packers in the emergency department. Hong Kong J Emerg Med 12: $112-118$

64. de Beer SA, Spiessens G, Mol W, Fa-Si-Oen PR, (2008) Surgery for body packing in the Caribbean: a retrospective study of 70 patients. World J Surg 32: 281-285

65. Schaper A, Hofmann R, Bargain P, Desel H, Ebbecke M, Langer C, (2007) Surgical treatment in cocaine body packers and body pushers. Int J Colorectal Dis 22: 1531-1535

66. Arora NP, Berk WA, Aaron CK, Williams KA, (2013) Usefulness of intravenous lipid emulsion for cardiac toxicity from cocaine overdose. Am J Cardiol 111: 445-447 